\title{
Sparsity-aware multiple relay selection in large multi-hop decode-and-forward relay networks
}

\author{
A. Gouissem ${ }^{1,2}$, R. Hamila1* ${ }^{*}$ N. Al-Dhahir ${ }^{3}$ and S. Foufou ${ }^{4}$
}

\begin{abstract}
In this paper, we propose and investigate two novel techniques to perform multiple relay selection in large multi-hop decode-and-forward relay networks. The two proposed techniques exploit sparse signal recovery theory to select multiple relays using the orthogonal matching pursuit algorithm and outperform state-of-the-art techniques in terms of outage probability and computation complexity. To reduce the amount of collected channel state information (CSI), we propose a limited-feedback scheme where only a limited number of relays feedback their CSI. Furthermore, a detailed performance-complexity tradeoff investigation is conducted for the different studied techniques and verified by Monte Carlo simulations.
\end{abstract}

Keywords: Large DF relay networks, Outage probability, MSE, Relay selection

\section{Introduction}

The basic concept behind cooperative communication can be traced back to [1], where the capacity of a three-node cooperative network was analyzed. Subsequent works proved in [2-5] that relaying techniques can achieve spatial diversity in wireless networks with only single-antenna nodes. This diversity can be achieved using a multitude of cooperative protocols and under different design criteria and channel information assumptions [6-11]. Among these protocols, two of the most widely used are the amplify-and-forward (AF) and decode-andforward (DF) protocols.

However, most of the cooperative protocols in the literature select only one relay to forward the data to the destination node [3]. The idea of single-relay selection was first generalized from dual-hop AF network to multiplerelay selection in [12]. Different schemes of multiple-relay selection have been also proposed $[7,8,13]$, where relays are assumed to cooperate either with full power or do not cooperate, i.e., no relay gain optimization is performed.

Furthermore, to satisfy the growing demand for highquality multimedia services in next-generation cellular

*Correspondence: hamila@qu.edu.qa

1 Electrical Engineering Department, Qatar University, Doha, Qatar

Full list of author information is available at the end of the article networks, high data rates have to be provided to the end users. Since mobile stations (MSs) are energy constrained devices, the transmit power of the MSs cannot be increased indiscriminately. Hence, the data rate can also be increased by reducing the distance between the source and destination node using multi-hop cellular networks whereby the base stations (BSs) would communicate with the faraway and otherwise unreachable MSs in multiple hops through intermediate relays [14-16].

However, when dealing with large DF relay networks [17], and especially in multi-hop cooperative networks [18-20], the relay selection process becomes highly complex. In fact, in many applications such as device-todevice (D2D) communication networks and wireless sensor networks, a large number of cooperating nodes are used, which leads to a dramatic increase in the complexity of the relay selection process.

Compressive sensing theory has been applied recently in the design of cognitive radio networks [21], finiteimpulse-response (FIR) linear equalizers [22], and relay selection schemes in dual-hop networks. In particular, the authors in [23] focus on multiple relay selection with a noisy limited feedback. In [24], a limited-feedback relay selection algorithm is also proposed and investigated for a multi-cast relay network. In addition, a compressive sensing (CS)-based relay selection algorithm that reduces the 
feedback overhead of relay networks under the assumption of noisy feedback channels is presented in [25]. In [26], a full-duplex relay-aided multi-user network is considered and the proposed scheme permits the BS to obtain channel state information (CSI) from a subset of strong users. CS is used also as joint source-channel coding (JSCC) for the source and the relays in multi-relay compressive cooperative schemes [27].

In this paper, we propose and investigate two multiple relay schemes for large multi-hop decode-and-forward relay networks. Our proposed schemes exploit the sparsity of the relay selection vector to select the relays by minimizing the mean-square error (MSE) based on a sparse signal recovery theory. We also propose and investigate a limited-feedback scheme in a multi-hop network, where the source collects only a limited amount of CSI to perform the selection.

In fact, when only few relays are selected from a large number of nodes, the relay selection vector becomes sparse. Hence, based on the sparse signal recovery theory, different signal recovery algorithms such as orthogonal matching pursuit (OMP) may be used to solve the relay selection problem $[17,28]$. Based on MSE minimization, we propose two different techniques for multiple-relay selection that reduce both the selection complexity and the outage probability compared with the existent techniques such as exhaustive search [12] and the selective decode and forward (SDF) technique [29] with and without power control.

The rest of this paper is organized as follows. In Section 2, the system model is presented. The proposed selection techniques are introduced and analyzed in Sections 3 and 4, respectively. In Section 5, the computational complexities of the different techniques are evaluated and compared. In Section 6, a new approach for a limited feedback is investigated. In Section 7, Monte Carlo simulations are carried out to confirm the analytical derivations and to demonstrate the higher efficiency of the proposed techniques compared to the conventional approaches. Finally, conclusions are drawn in Section 8.

\section{System model}

In this paper, the adopted system model consists of one source (S), $M$ equidistant clusters of $N$ relays and one destination (D) as shown in Fig. 1. We define also the total number of clusters including the source (cluster 0 ) and the destination (cluster $M+1$ ) as $N_{c}=M+2$.

For each $i \in\{1 . . N\}$ and $j \in\{1 . . M\}, R_{i, j}$ denotes the $j$ th relay in the $i$ th cluster. All nodes are equipped with a single antenna which can be used in a half-duplex mode for both transmission and reception. The direct link between the source and the destination is ignored due to its assumed large path loss.

The data is transmitted from the source to the destination using $K$ different paths based on a decode-andforward technique. Different selection schemes are proposed in this paper where the paths may be selected directly by the source or at each stage by the nodes forwarding the data.

In addition, each node is assumed to have perfect CSI knowledge of its last hop links. This CSI is used to decode the received signal in the intermediate hops and to combine the different received versions of the signal using maximum ratio combining (MRC) at the destination.

Denote the channel coefficient from a node $A$ to a node $B$ as $h_{A, B}$, which is assumed to follow complex Gaussian distribution with mean zero and variance $\sigma_{A, B}^{2}$. We assume also that these channels are flat-fading and remain constant during the transmission.

Moreover, to analyze the effect of the relay's positions, we adopt an asymmetric network geometry where the distance from any node in cluster $i$ to any node in cluster $i+1$ is equal to $d_{i}$. Without loss of generality, the ditance between the source and the destination is normalized to be equal to 1 [30], i.e., $\sum_{i=0}^{M} d_{i}=1$.

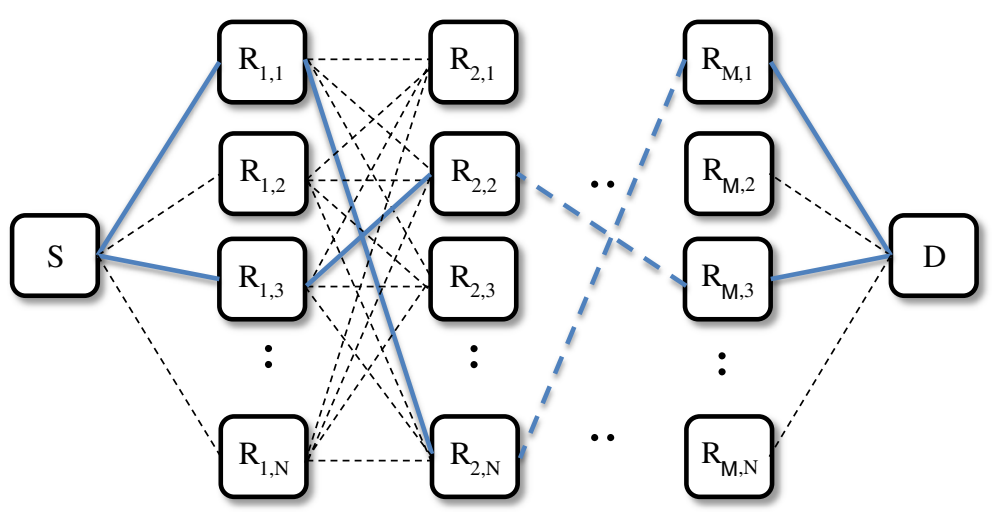

Fig. 1 System model 
In the subsequent numerical analysis, the path-loss exponent $\alpha$ is assumed to be equal to 2 . Hence, the variance of the complex Gaussian distribution corresponding to the channel linking a node $A$ in cluster $i$ with any node $B$ in cluster $i+1$ is expressed by

$$
\sigma_{A, B}^{2}=E\left(\left|h_{A, B}\right|^{2}\right)=\left(\frac{d_{S, D}}{d_{A, B}}\right)^{2}=\frac{1}{d_{i}^{2}} \triangleq \sigma_{i}^{2},
$$

where $E($.$) denotes the expectation of a random variable$ and the distance between the node $A$ and the node $B$ is denoted by $d_{A, B}$.

Considering the link $A \rightarrow B$ at any transmission stage where node $B$ receives from node $A$, the signal denoted by $y_{A, B}$ is given by

$$
y_{A, B}=h_{A, B} x_{A}+n_{A, B},
$$

where $x_{A}$ denotes the transmitted signal by node $A$ and $n_{A, B}$ is the additive white Gaussian noise (AWGN) on the $\operatorname{link} A \rightarrow B$.

Next, we propose two techniques to select the relays that will decode and forward the data.

- EndtoEnd relay selection scheme which selects the best $K$ paths to forward the data directly by the source

- PerGroup relay selection scheme which divides the network into different groups and the best $K$ paths selections are performed independently for each group

\section{EndtoEnd relay selection scheme \\ 3.1 Motivation}

As stated in Section 2, $K$ different paths are selected to forward the data from the source to the destination. Then, the different received signals are combined using MRC to reconstruct the original data at the destination. We consider first the received signal through one selected path $P \triangleq\left[S \rightarrow R_{1}^{P} \rightarrow R_{2}^{P} . . \rightarrow . . D\right]$, where $R_{i}^{P}$ refers to the relay in cluster $i$ that belongs to the path $P$.

Since DF is used, the equivalent signal-to-noise ratio (SNR) for the relay network composed by only the path $P$ can be approximated by $\gamma_{\mathrm{eq}}^{P}=\min \left(\gamma_{1}^{P} ; \ldots \gamma_{M+1}^{P}\right)$, where $\gamma_{i}^{P}$ denotes the SNR of the link corresponding to the $i$ th hop in $P$ [31].

Hence, for each selected path $P$, the transmission becomes equivalent to a transmission over a single link with an equivalent SNR

$\gamma_{\mathrm{eq}}^{P}=\min \left(\gamma_{S, R_{1}^{P}} ; \gamma_{R_{1}^{P}, R_{2}^{P}} ; . . ; \gamma_{R_{M}^{P}, D}\right)$, where $\gamma_{A, B}=$ $\frac{\left|h_{A, B}\right|^{2}}{N_{0}}$ and $N_{0}$ denotes the noise spectral density.

In this proposed scheme, we force all the relays in one selected path $P$ to use the same gain factor. In fact, a set of $N_{\text {Paths }}^{E t E} \triangleq N^{M}$ possible paths is present for possible selections. Therefore, we define the relay selection vector $\mathbf{g}_{\mathbf{s}} \triangleq\left[\mathbf{g}_{\mathbf{s}}(1) \ldots \mathbf{g}_{\mathbf{s}}\left(N_{\text {Paths }}^{E t E}\right)\right]^{T}$, where for each $P \in \mathbb{P} \triangleq\{$ set of all $N_{\text {Paths }}^{E t E}$ possible paths $\}, \mathbf{g}_{\mathbf{s}}(P)$ denotes the selected gain factor for all the relays in the path $P$. Consequently, the equivalent SNR for the path $P$ becomes

$$
\begin{aligned}
\gamma_{\mathrm{eq}}^{P} & =\min \left(\left|\mathbf{g}_{\mathbf{s}}(P)\right|^{2} \gamma_{S, R_{1}^{P}} ;\left|\mathbf{g}_{\mathbf{s}}(P)\right|^{2} \gamma_{R_{1}^{P}, R_{2}^{P}} ; . . ;\left|\mathbf{g}_{\mathbf{s}}(P)\right|^{2} \gamma_{R_{M-1}^{P}, D}\right) \\
& =\left|\mathbf{g}_{\mathbf{s}}(P)\right|^{2} \min \left(\gamma_{S, R_{1}^{P}} ; \gamma_{\left.R_{1}^{P}, R_{2}^{P} ; . . ; \gamma_{R_{M-1}^{P}, D}\right)}\right. \\
& =\left|\mathbf{g}_{\mathbf{s}}(P)\right|^{2} \frac{\left|\min \left(h_{S, R_{1}^{P}} ; h_{R_{1}^{P}, R_{2}^{P}} ; . . ; h_{R_{M-1}^{P}, D}\right)\right|^{2}}{N_{0}}
\end{aligned}
$$

Consequently, we can also approximate the received signal at the destination through a path $P$, denoted by $y_{D}^{P}$, by the signal that passes through a channel with the same characteristics of the adopted system model (zeromean complex Gaussian) and with the same equivalent received SNR in Eq. (3) and thus also the same outage probability, i.e,

$$
y_{D}^{P} \approx \mathbf{g}_{\mathbf{s}}(P) h_{\mathrm{eq}}^{P} x+\hat{n},
$$

where $\hat{n}$ is an AWGN with variance $N_{0}$ and $h_{\text {eq }}^{P}=$ $\min \left(h_{S, R_{1}^{P}}, h_{R_{1}^{P}, R_{2}^{P}}, . ., h_{R_{M}^{P}, D}\right)$. Consequently, the combined received signal becomes [17]:

$$
y_{D} \approx \mathbf{g}_{\mathbf{s}}{ }^{H}(\mathbf{h} x+\mathbf{v})
$$

where $\mathbf{v}$ is an $N_{\text {Paths }}^{E t E} \times 1$ AWGN vector with variance $N_{0}$, $\mathbf{h}$ is an $N_{\text {Paths }}^{E t E} \times 1$ equivalent channel vector defined by $\mathbf{h}=\left[h_{\mathrm{eq}}^{1}, h_{\mathrm{eq}}^{2}, . ., h_{\mathrm{eq}}^{N_{\text {Paths }}^{E t E}}\right]^{T}$.

\subsection{Relay selection}

To perform the relay selection, we assume that all the CSI is known by the source. We understand that estimating all the CSI by the source is a complicated task that may induce transmission delays. However, if the channels are slowly varying, less feedback is required to estimate the CSI. Furthermore, based on sparse signal recovery, the amount of CSI feedback can be dramatically reduced as shown in $[32,33]$. In addition, this problem is solved by our limited-feedback scheme proposed and investigated in Section 7 where we show that almost the same outage performance can be reached with a limited CSI feedback.

The relay selection vector $\mathbf{g}_{\mathbf{s}}$ is computed based on the MSE minimization. From Eq. (5), we deduce that the error signal can be expressed as follows

$$
e=x-y_{D}=x-\mathbf{g}_{\mathbf{s}}{ }^{H}(\mathbf{h} x+\mathbf{v})
$$

Hence, the MSE at the destination is given by

$$
\begin{aligned}
E\left[|e|^{2}\right] & =\sigma_{x}^{2}-\mathbf{g}_{\mathbf{s}}{ }^{H} \mathbf{h} \sigma_{x}^{2}-\mathbf{g}_{\mathbf{s}}{ }^{H} \sigma_{x}^{2}+\mathbf{g}_{\mathbf{s}}{ }^{H}\left(\sigma_{x}^{2} \mathbf{h h}^{H}+\mathbf{R}_{\mathbf{v v}}\right) \mathbf{g}_{\mathbf{s}} \\
& =\sigma_{x}^{2}-\mathbf{g}_{\mathbf{s}}{ }^{H} \tilde{\mathbf{h}}-\tilde{\mathbf{h}}^{H} \mathbf{g}_{\mathbf{s}}+\mathbf{g}_{\mathbf{s}}{ }^{H} \mathbf{R g}_{\mathbf{s}}
\end{aligned}
$$


where

$$
\begin{aligned}
\tilde{\mathbf{h}} & =\mathbf{h} \sigma_{x}^{2} \\
\mathbf{R} & =\sigma_{x}^{2} \mathbf{h} \mathbf{h}^{H}+\mathbf{R}_{\mathbf{v v}} \\
\mathbf{R}_{\mathbf{v v}} & =E\left(\mathbf{v} \mathbf{v}^{H}\right) \\
\sigma_{x}^{2} & =E\left(|x|^{2}\right) .
\end{aligned}
$$

Considering the Cholesky factorization of the positive denite matrix $\mathbf{R}=\mathbf{L} \mathbf{L}^{H}$ where $\mathbf{L}$ is an $N_{\text {Paths }}^{E t E} \times N_{\text {Paths }}^{E t E}$ lowertriangular matrix, we can rewrite Eq. (8) as follows

$$
\text { MSE }=\sigma_{x}^{2}-\mathbf{g}_{\mathbf{s}}{ }^{H} \mathbf{L} \mathbf{L}^{-1} \tilde{\mathbf{h}}-\tilde{\mathbf{h}}^{H} \mathbf{L}^{-H} \mathbf{L}^{H} \mathbf{g}_{\mathbf{s}}+\mathbf{g}_{\mathbf{s}}{ }^{H} \mathbf{L} \mathbf{L}^{H} \mathbf{g}_{\mathbf{s}}
$$

By completing the square in Eq. (9), we get

$$
\operatorname{MSE}=\sigma_{x}^{2}-\tilde{\mathbf{h}}^{H} \mathbf{L}^{-H} \mathbf{L}^{-1} \tilde{\mathbf{h}}+\left\|\mathbf{L}^{H} \mathbf{g}_{\mathbf{s}}-\mathbf{L}^{-1} \tilde{\mathbf{h}}\right\|_{2}^{2}
$$

For the case where no power control is used (the vector $\mathbf{g}_{\mathbf{s}}$ identifies only which paths to be used without any information about transmission power), it is obvious that the optimal solution of Eq. (10) can be found by an exhaustive search among all the possibilities of the selected paths [12]. However, as it will be discussed in Section 5, this task requires a high computation complexity. Hence, to simultaneously reduce the complexity and select a complex vector $\mathbf{g}_{\mathbf{s}}$ instead of a Boolean one, we use sparse signal recovery techniques. In fact, since only a small number of paths $K$ is selected, the vector $\mathbf{g}_{\mathbf{s}}$ becomes sparse ${ }^{1}$ which can be exploited to reduce the complexity by sparse signal recovery algorithms and we adopt the OMP algorithm in this paper for its simplicity.

Note that in EndtoEnd, $K$ paths out of $\left.N^{(} M-1\right)$ are selected. Consequently, even if a large number of relays $K$ is selected (up to $N$ ), the selection vector $\mathbf{g}_{\mathbf{s}}$ will remain sparse by network design and can be computed using OMP.

The expression of the MSE in Eq. (10) is divided into the following two terms:

$$
\begin{aligned}
\mathrm{MSE}_{\min } & =\sigma_{x}^{2}-\tilde{\mathbf{h}}^{H} \mathbf{L}^{-H} \mathbf{L}^{-1} \tilde{\mathbf{h}} \\
\mathrm{MSE}_{\text {excess }} & =\left\|\mathbf{L}^{H} \mathbf{g}_{\mathbf{s}}-\mathbf{L}^{-1} \tilde{\mathbf{h}}\right\|_{2}^{2}
\end{aligned}
$$

Since $\mathrm{MSE}_{\min }$ does not depend on $\mathbf{g}_{\mathbf{s}}$, the MSE is minimized by minimizing the term $\mathrm{MSE}_{\text {excess }}$ which can be controlled through the path selection vector $\mathbf{g}_{\mathbf{s}}$. As in [17], to select multiple paths which minimize MSE from Eq. (10), we use the OMP algorithm which proceeds by finding, in each iteration, one column of the matrix $\mathbf{L}^{H}$ which is the most correlated with the residual error vector obtained by subtracting the contributions of the selected paths in the previous iteration from the vector $\mathbf{L}^{-1} \tilde{\mathbf{h}}$, then by solving a least squares problem to obtain a new signal estimate and updating the new set of possible paths.

We adopt the OMP algorithm in [28] for which the stopping criterion is the desired number of selected paths $K$ denoted by

$$
\mathbf{g}_{\mathbf{s}}=\operatorname{OMP}\left(\mathbf{L}^{H}, \mathbf{L}^{-1} \tilde{\mathbf{h}}, K_{\text {iterations }}\right)
$$

In fact, in each of the $K$ OMP iterations, the path that minimizes $\mathrm{MSE}_{\text {excess }}$ is selected until finally obtaining $K$ paths.

\subsection{Independent and dis-joint selection}

For the EndtoEnd relay selection scheme, selecting $K$ out of $N_{\text {Paths }}^{E t E}$ paths to forward the data to the destination may result in using the same relay in different paths. This should increase the diversity of the system. However, in practice, it may introduce some implementation challenges since the same relay may have to receive different signals from different sources at the same time. Therefore, to deal with these challenges, we propose and differentiate in this section between two different versions of EndtoEnd relay selection scheme.

\subsubsection{Independent selection}

When independent selection is performed, OMP algorithm is used such that, at each iteration, one column of the matrix $\mathbf{L}^{H}$ is selected based only on the updated residual error. Hence, the same relay may be selected simultaneously in multiple paths. Hence, further diversity is introduced to the system since channels with high SNR may be used multiple times to improve the outage performance of the system.

However, in practice, this may introduce some implementation challenges since the same relay may have to receive different signals from different sources at the same time. Furthermore, the same relay may have to transmit different data to different relays at the same time. Thus, this might be possible only if orthogonal channels are used based on time division multiplexing (TDM) or frequency division multiplexing (FDM).

At the $i$ th iteration (where, $i \in\{1 . . K\}$ ) of the OMP recovery algorithm, one path is selected between the possible remaining $N_{\text {Paths }}^{E t E}-i+1=N^{M}-i+1$ columns of the matrix $\mathbf{L}^{H}$ based on the MSE residual error.

\subsubsection{Dis-joint selection}

When dis-joint selection is performed, the OMP algorithm is forced at each step to select one of the paths for which all the relays are never used. Hence, the multiple receptions or transmissions problem for the same relay is solved since all the selected paths are dis-joint. In particular, in step 6 of the OMP algorithm in [28], we remove from the set of indices available for selection the indices of all the paths that have joint relays with the last selected path.

Thus, the selection complexity is reduced compared to the joint selection scheme since the number of possible paths reduces dramatically at each iteration. In fact, at the iteration $i \in\{1 . . K\},(N-i+1)^{M}$ columns are available in 
$\mathbf{L}^{H}$ for possible selection instead of $\left(N^{M}-i+1\right)$ in joint selection EndtoEnd selection scheme.

\section{PerGroup selection scheme}

\subsection{Motivation}

In the EndtoEnd selection scheme, the source is assumed to know all the CSI to perform the path selection. However, this might be difficult to implement in practice especially in large relay networks. Furthermore, the number of possible paths $N_{\text {Paths }}=N^{M}$ is very high which results in a high selection complexity. This problem may be solved using the sparse signal recovery theory to reduce the CSI feedback by allowing only relays that have an SNR higher than a certain threshold to send their CSI to the source as in [32,33]. However, in this section, we propose another solution which consists of dividing the network into independent groups for which the path selections are performed independently from each other.

In the PerGroup selection scheme, the network is divided into $N_{G}$ groups of $N_{C}^{p G} \triangleq 1+$ floor $\left[\frac{M+1}{N_{G}}\right]$ clusters, where floor $[x]$ denotes the largest integer less than or equal to $x$.

As it can be seen in Fig. 2 (where $M=5, N_{G}=3$ and $N_{C}^{p G}=3$ ), each group consists of 1 (in the first group) or $K$ sources (other groups) and $N_{C}^{p G}-2=$ floor $\left[\frac{M+1}{N_{G}}\right]-1$ clusters of relays.

The source and the intermediate $K$ relays at the beginning of each group are assumed to have perfect CSI knowledge for all the links in the corresponding group.

\subsection{Relay selection}

Concerning the relay selection in the first group, the source has to select $K$ out of $N_{\text {Paths }}^{p G \mid p S} \triangleq N^{N_{C}^{p G}-1}$ possible paths to forward the data to the next group. Consequently, we define a virtual destination $D_{1}$ at the end of group 1 able to combine all the received signals using $\mathrm{MRC}^{2}$. The received signal at this virtual destination can be approximated by

$$
y_{D_{1}} \approx \mathbf{g}_{\mathbf{s}}^{\mathbf{1}^{H}}\left(\mathbf{h}_{\mathbf{1}} x+\mathbf{v}\right)
$$

where $\mathbf{v}$ is an $N_{\text {Paths }}^{p G \mid p S} \times 1$ AWGN vector with variance $N_{0}$ and $\mathbf{h}_{\mathbf{1}}$ is an $N_{\text {Paths }}^{p G \mid p S} \times 1$ equivalent channel vector defined by $\mathbf{h}_{\mathbf{1}}=\left[h_{1}^{1}, h_{1}^{2}, . ., h_{1}^{N_{\text {Paths }}^{p G \mid p S}}\right]^{T}$, where for each path, $P \in \mathbb{P} \triangleq\left\{1, . ., N_{\text {Paths }}^{p G \mid p S}\right\}, h_{1}^{P}=\min \left(h_{S, R_{1}^{P}} ; h_{R_{1}^{P}, R_{2}^{P}}\right.$ $\left.;. . ; h_{R^{P}{ }_{C}^{p G}, R^{P}}{ }_{N_{C}^{p G}-1}^{p G}\right)$.

Hence, and by analogy with the EndtoEnd selection scheme, the OMP algorithm is used to compute the gain selection vector of the first group $\mathbf{g}_{\mathbf{s}}^{\mathbf{1}}$ by replacing $\mathbf{h}$ by its new expression. Also, similarly to Section 3, both the disjoint and indepedndent selection schemes are considered in the PerGroup selection technique.

Concerning any other group $G$, its first cluster contains always $K$ relays holding the data forwarded by the previous group which are denoted by $\left\{\tilde{R}_{i} \mid i=1 . . K\right\}$, respectively. By assuming that the group $G-1$ forwarded approximately the same data signal $\tilde{x}$ to the $K$ relays in the first cluster of the group $G$, this group becomes equivalent to a system with only one virtual source $S_{2}$ instead of $K$ sources and one virtual destination $D_{2}$ that combines all the received signals as explained in Fig. 2. In fact, the virtual source $S_{2}$ is connected to $K$ duplicates of the following clusters by the channels connecting $\tilde{R}_{i}$ to the next clusters where $i \in\{1, . . K\}$ (Fig. 3).

Consequently, each one of the intermediate groups contains $N_{\text {Paths }}^{p G} \triangleq K N_{\text {Paths }}^{p G \mid p S}=K \cdot N^{N_{C}^{p G}-1}$ possible paths, and the combined signal by the virtual destination $D_{G}$ can be approximated by

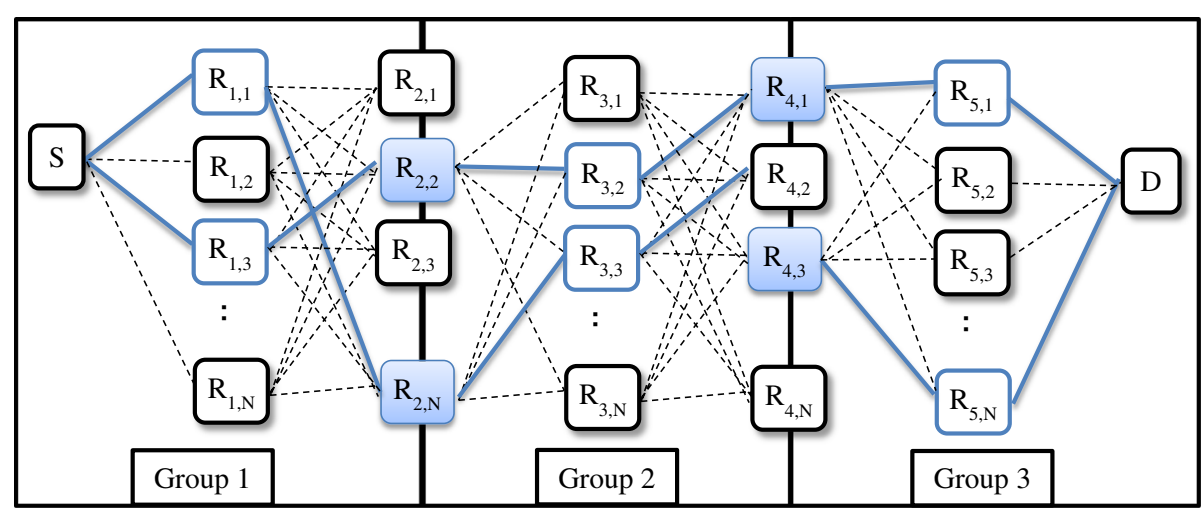

Fig. 2 Network division into independent groups in the PerGroup selection technique 


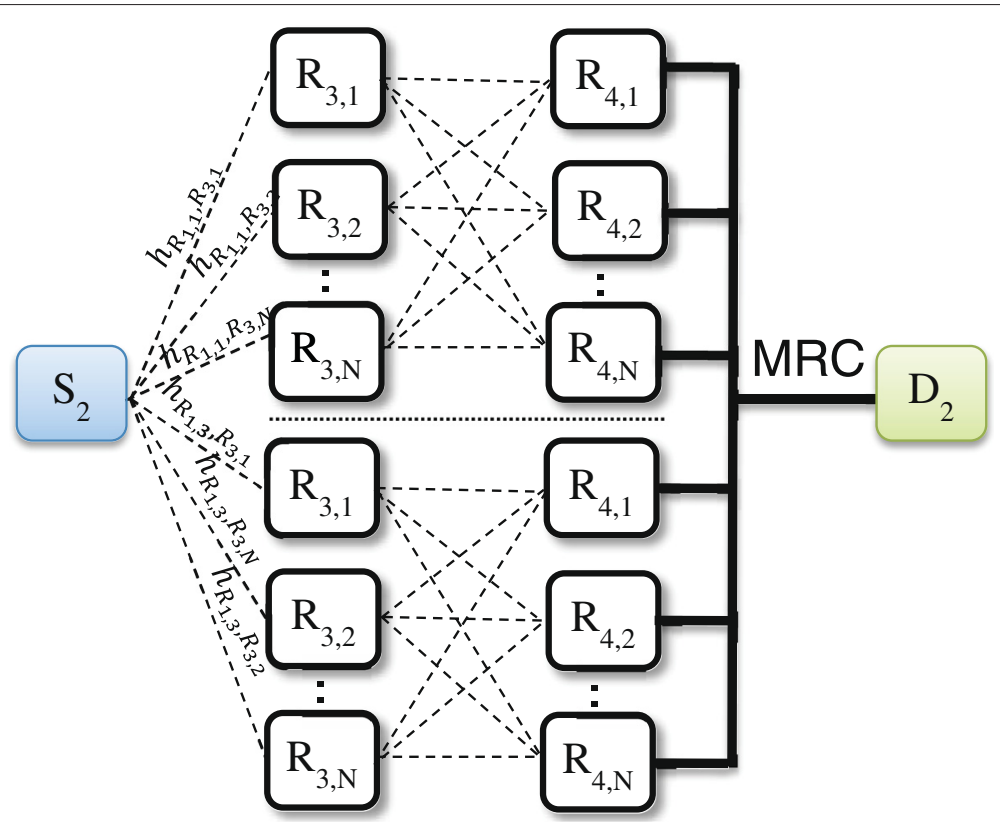

Fig. 3 Equivalent network model for the second group

$$
\mathbf{y}_{\mathbf{D}_{\mathbf{G}}} \approx \mathbf{g}_{\mathbf{s}}^{\mathbf{G}^{H}}\left(\mathbf{h}_{\mathbf{G}} \tilde{x}+\mathbf{v}\right)
$$

where $\mathbf{v}$ is an $N_{\text {Paths }}^{p G} \times 1$ AWGN vector with variance $N_{0}$ and $\mathbf{h}_{\mathbf{G}}$ is an $N_{\text {Paths }}^{p G} \times 1$ equivalent channel vector defined by $\mathbf{h}=\left[\mathbf{h}^{\mathbf{1}^{T}}\left|\mathbf{h}^{\mathbf{2}^{T}}\right| . . \mid \mathbf{h}^{\mathbf{K}^{T}}\right]^{T}$, where for each $i \in\{1 . . K\}, \mathbf{h}^{\mathbf{i}}$ refers to the equivalent channel when the source is $\tilde{R}_{i}$. This equivalent channel is expressed by $\mathbf{h}^{\mathbf{i}}=$ $\left[\mathbf{h}_{\mathbf{i}}^{\mathbf{1}}, \mathbf{h}_{\mathbf{i}}^{\mathbf{2}}, . ., \mathbf{h}_{\mathbf{i}}^{\mathbf{N}_{\text {Paths }}^{\mathbf{p G} \mid \mathbf{p S}}}\right]^{T}$, where for each path $P \in \mathbb{P}, \mathbf{h}_{\mathbf{i}}^{\mathbf{P}}=$

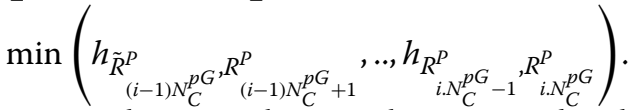

Note that to make sure that every relay that received a data forwards it, we force the OMP algorithm to select at each iteration one path referring to a different relay $\tilde{R}_{j}$. In fact, at the iteration $i \in\{1 . . K\},(K-i+1) \cdot N_{\text {Paths }}^{p G \mid p S}=$ $(K-i+1) \cdot N^{N_{C}^{p G}-1}$ columns are available in $\mathbf{L}^{H}$ for possible selection.

In the PerGroup scheme, all the CSI is assumed to be known at the source (or in the $K$ corresponding relays in the first cluster of the intermediate groups). We also proposed a new limited-feedback scheme that makes the source use only partial knowledge of the CSI. We showed that it is better in terms of outage probability to get full CSI especially when $N_{c p g}$ is small. However, in terms of network throughput, it is better to use a limited feedback since the gain from the CSI knowledge becomes at some point not enough to compensate for the losses in terms of the time needed to collect the CSI.

Concerning the selection criteria of $K$, there is no optimal choice of $K$ because of the following:

1. In multiple relay selection schemes and when sparse signal recovery theory is not used, it is always better to use more relays to achieve better outage performance. However, using all the relays of the network to forward the data is not practical and may lead to network congestion. Hence, the optimal $K$ is the total number of relays, but in practice, $K$ is chosen by the network administrator to make a compromise between the desired outage performance and network congestion.

2. In the PerGroup scheme, $K$ paths out of $N^{\left(N_{c p g}-1\right)}$ (or $K N^{\left(N_{c p g}-2\right)}$ in the last group) are selected in each group, where $N$ and $N_{c p g}$ denote the number of relays per cluster and number of clusters PerGroup, respectively. Consequently, even if a large number of relays $K$ is selected (up to $N$ ), the selection vector $\mathbf{g}_{\mathbf{s}}$ will remain sparse and the can be computed using OMP.

3. The sensing matrix is a square full-rank matrix as in [17]. Note that even though the sensing matrix is full-rank and the selection problem is well-posed, the optimal solution is not sparse (all the paths are selected) and OMP is used to compute the "best" $K$ sparse solution. 


\section{Computation complexity comparison}

\subsection{EndtoEnd independent relay selection}

\subsubsection{Exhaustive search}

For the case where $g_{s}$ is a Boolean vector (no power control ), the optimal EndtoEnd selection can be obtained by exhaustive search where there are $\left(\begin{array}{l}K \\ N_{\text {Paths }}\end{array}\right)=$ $O\left(\left(N^{M-1}\right)^{K}\right)$ possibilities. To select the best one of them, the complexity of the exhaustive search becomes:

$$
C_{E t E-I}^{E S}=O\left(\left(N^{M}\right)^{K}\right)
$$

\subsubsection{Proposed model}

In the OMP-based proposed technique, $K$ out of the $N_{\text {Paths }}$ paths are selected independently. By computing a sparse vector containing $K$ non-zero elements based on a measurement vector of length $N_{\text {Paths }}$ using OMP. Hence, the computation complexity can be expressed by [17].

$$
C_{E t E-I}^{P}=O\left(K^{2} N^{M}\right)
$$

\subsection{EndtoEnd dis-joint relay selection}

\subsubsection{Exhaustive search}

When dis-joint relay selection is performed based on ES, the number of possible combinations of paths is dramatically reduced compared to independent selection scheme since the paths have to be dis-joint and not containing any common relay. Hence, we deduce that the computation complexity for the EndtoEnd dis-joint ES technique is very small compared to the EndtoEnd ES independent technique.

$$
C_{E t E-D}^{E S}<<C_{E t E-I}^{E S}
$$

\subsubsection{Proposed model}

When dis-joint relay selection is performed based on OMP, at each selection iteration $i$, the number of possible paths for the selection are reduced from $N^{M}-i+1$ to $(N-i+1)^{M}$ compared with the independent selection model. Hence, we deduce that the computation complexity for the EndtoEnd dis-joint OMP technique is very small compared to the EndtoEnd independent OMP technique.

$$
C_{E t E-D}^{P}<<C_{E t E-I}^{P}
$$

\subsection{PerGroup relay selection}

\subsubsection{Exhaustive search}

In the PerGroup relay selection technique, the best $K$ paths for each group of $N_{C}^{p G}$ are selected. Hence, if independent selection is performed with the ES algorithm, the computation complexity would be

$$
\tilde{C}_{p G}^{E S}=O\left(N_{G}\left(N^{\text {floor }\left[1+\frac{M+1}{N_{G}}\right]}\right)^{K}\right)
$$

Hence, we deduce that when dis-joint selection is performed, the computation complexity becomes

$$
\left.C_{p G}^{E S}<<O\left(N_{G}\left(N^{\text {floor }\left[1+\frac{M+1}{N_{G}}\right]}\right)\right)^{K}\right)
$$

\subsubsection{Proposed model}

In the PerGroup relay selection technique, the best $K$ paths for each group of $N_{C}^{p G}$ are selected. Hence, if independent selection is performed with the OMP algorithm, the computation complexity would be

$$
\tilde{C}_{P G}^{P}=O\left(N_{G} K^{2} N^{\text {floor }\left[1+\frac{M}{N_{G}}\right]}\right) .
$$

Hence, we deduce that when dis-joint selection is performed, the computation complexity becomes

$$
C_{P G}^{P}<<O\left(N_{G} K^{2} N^{\text {floor }\left[1+\frac{M}{N_{G}}\right]}\right)
$$

\section{Limited-feedback scheme}

To reduce the computation complexity and also the amount of CSI feedback needed for the relays selection for both the EndtoEnd and PerGroup schemes, we investigate in this section a limited-feedback model where the source (or intermediate sources) no longer has full CSI knowledge.

As stated in Section 2, each node is assumed to have perfect knowledge of the CSI corresponding to the links with the relays of the previous cluster. Thus, to limit the amount of CSI feedback, a limited number of nodes are allowed to feedback their CSI to the previous cluster. This is done if the relay already received the CSI from at least one node of the next cluster and if the known SNR for the previous hop is above the feedback threshold. The feedback threshold $\gamma_{\text {th }}$ is optimized to meet a target feedback percentage level.

In particular, for a node $\mathrm{A}$ in cluster $i$, the probability that $A$ has a link with the next cluster with an SNR above $\gamma_{\text {th }}$ is given by

$$
\begin{aligned}
P_{i} & =F_{i}\left(\gamma_{\text {th }}\right) \\
& =1-\exp \left(-\lambda_{i} \gamma_{\text {th }}\right),
\end{aligned}
$$

where $F_{i}$ denotes the cumulative density function (CDF) of the channel realizations corresponding to the links in hop $i\left(h_{i}\right), \lambda_{i}=\frac{1}{\sigma_{i}^{2}}$.

For each possible path from the source to the destination, if the SNR in any intermediate hop is below $\gamma_{\text {th }}$, then the source will not receive its CSI. Hence, the fraction of CSI that would be received (in average) by the source is given by 


$$
r=1-\prod_{i=1}^{M-2}\left(1-P_{i}\right) \quad=1-\exp \left(-\sum_{i=1}^{M-2} \lambda_{i} \gamma_{\text {th }}\right)
$$

Consequently,

$$
\begin{aligned}
1-r & =\exp \left(-\sum_{i=1}^{M-2} \lambda_{i} \gamma_{\mathrm{th}}\right), \\
\log (1-r) & =-\sum_{i=1}^{M-2} \lambda_{i} \gamma_{\mathrm{th}} \\
\gamma_{\mathrm{th}} & =\frac{-\log (1-r)}{\sum_{i=1}^{M-2} \lambda_{i}}
\end{aligned}
$$

For the PerGroup scheme, by analogy, we get the following expression for the threshold

$$
\gamma_{\mathrm{th}}=\frac{-\log (1-r)}{\sum_{i \in S} \lambda_{i}}
$$

where $S$ denotes the set of clusters in the group for which the selection is done.

A limited number of paths $K$ is selected (up to $N$ ). As it can be seen in Fig. 8, an EndtoEnd scheme using a limited feedback of only $20 \%$ (the source knows the CSI of only $20 \%$ of the paths) has the same outage performance as a system with full CSI knowledge for the EndtoEnd scheme (when $K=2, N=10, N_{c}=5$ ). Hence, the amount of feedback can be dramatically reduced without affecting the outage performance.

\section{Simulation results}

In this section, the simulated performance of the proposed schemes is evaluated in terms of outage probability, bit error rate, and network throughput.

A symbol is considered in outage if it is received at the destination with an equivalent end-end SNR below a predefined threshold $\epsilon$ [34]. Since MRC is used at the destination, the equivalent end-end SNR of the combined received signal is the sum of the end-end SNRs through the $K$ used paths.

When computing the network throughput, the amount of air-time reserved for feedback is no longer negligible as compared to the transmission time. Hence, we define the achievable throughput as the number of transmitted bits per unit time $(\mathrm{bps} / \mathrm{Hz})$. The network throughput is explicitly given by [26]

$$
\begin{aligned}
T & =\left(\frac{T_{c}-N_{p} T_{m s}}{T_{c}}\right) \log _{2}\left(1+\gamma_{e}\right) \\
& =\left(1-N_{P} \tau\right) \log _{2}\left(1+\gamma_{e}\right)
\end{aligned}
$$

where $\gamma_{e}$ denotes the equivalent MRC received SNR as detailed in Section 3, $T_{c}$ is the channel coherence time,
$T_{m s}$ is the time needed to transmit one feedback, $\tau=\frac{T_{m s}}{T_{c}}$, and $N_{p}$ is the number of CSI that need to be collected. Note that when limited feedback is used, the amount of CSI that need to be collected becomes $N_{p}=r N_{p}^{t}$, where $r$ denotes the ratio of CSI that need to be collected and $N_{p}^{t}$ denotes the total number of possible paths. To have more insight, we simulate the network throughput for $\tau=$ $1 / 600$ [26].

We also compare these results with previously proposed techniques. In fact, to the authors' knowledge, no previous work has been done for multiple relay selection in multi-hop DF network. However, the authors in [29], proposed two multiple relay selection schemes for dual-hop cooperative networks entitled SDF and SDF with power control. Hence, and for a fair comparison with our multihop schemes, the selection process for these techniques is repeated every two hops as detailed for the PerGroup selection scheme. We also adapted the ES technique in [12] to our system model by making an exhaustive search over all the possible EndtoEnd paths or over all the possible paths in each group. In addition, for fair comparison, the same average transmit power per relay constraint is assumed, i.e., the selection vector $g_{s}$ satisfies $E\left(g_{s}^{H} g_{s}\right)=K$ for all the studied techniques. The simulation parameters used in the simulations are shown in Table 1.

Figure 4 compares the outage performance of the Per$\operatorname{Group}\left(n_{G}=2,3\right)$ and the EndtoEnd schemes with ES and SDF (with and without power control) techniques when the number of clusters is $N=7, K=2$ relays are selected out of $N=5$ at each hop. It can be seen that the bigger the number of groups is, the higher is the outage probability. In fact, it is always better to select based on the CSI of all the hops but at the cost of more CSI that needs to be collected and higher computation complexity. Also, when applying independent selection, the outage probability is always better than dis-joint selection model since it achieves more selection diversity, but at the price of some practical implementation issues that may be caused by allowing one relay receiving (or sending) from (to) different nodes and also higher computation complexity.

It can be seen that the proposed algorithm outperforms the existing techniques in terms of outage probability. In

Table 1 Simulation parameters

\begin{tabular}{lll}
\hline Parameter & Value & Description \\
\hline$\epsilon$ & 3 & Outage probability threshold \\
$N$ & $\{5,6,8\}$ & Number of relays per cluster \\
$K$ & $\{2 \rightarrow 5\}$ & number of selected relays \\
$d$ & Random vector & Normalized distance between clusters \\
$N_{G}$ & $1 . .3$ & Number of groups \\
$N_{C}$ & $3,5,7$ & Number of clusters \\
SNR & $\{0 \rightarrow 30 \mathrm{~dB}\}$ & Signal-to-noise ratio per hop \\
\hline
\end{tabular}




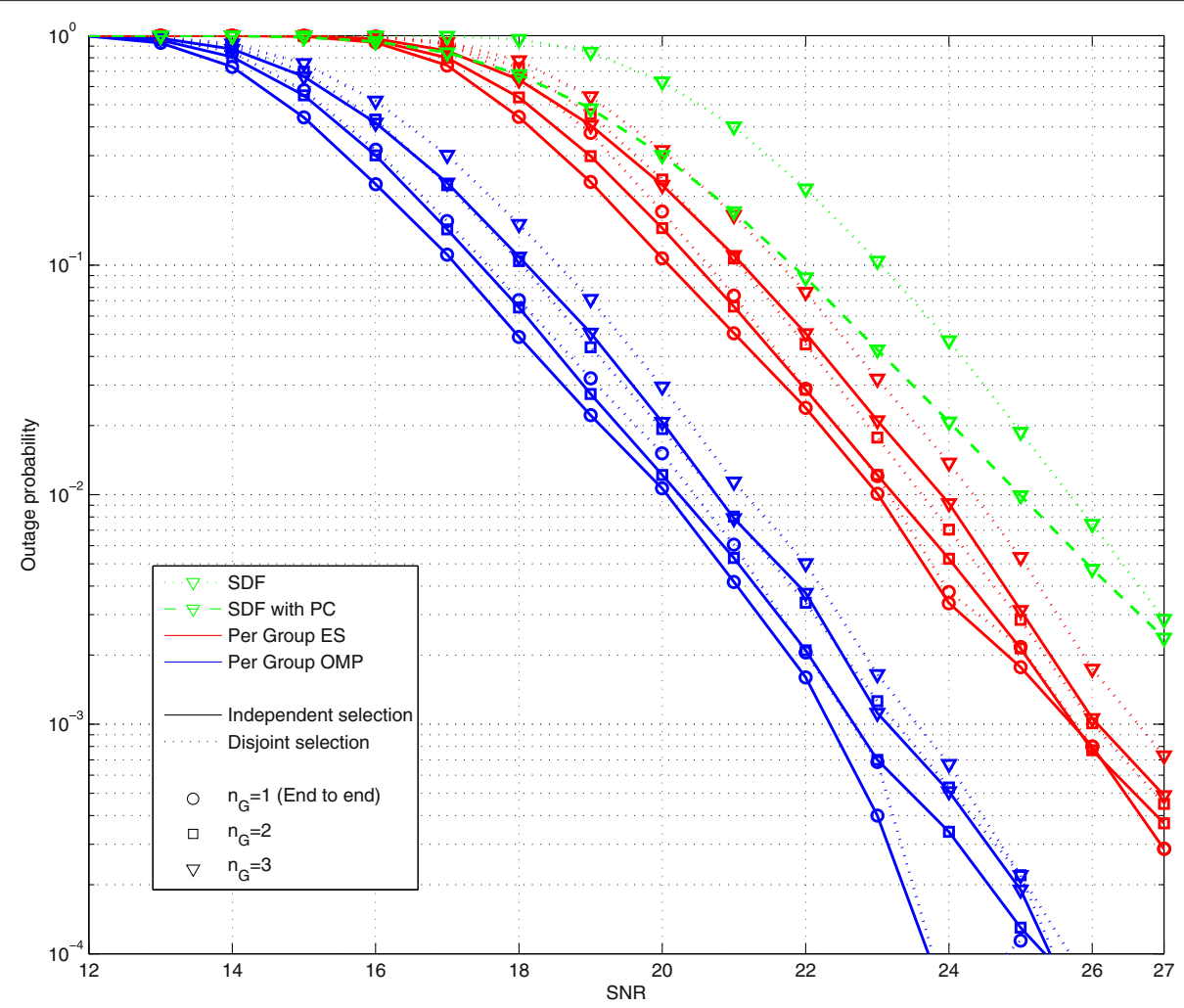

Fig. 4 The effect of the number groups in the outage performance of the PerGroup scheme compared to ES and SDF

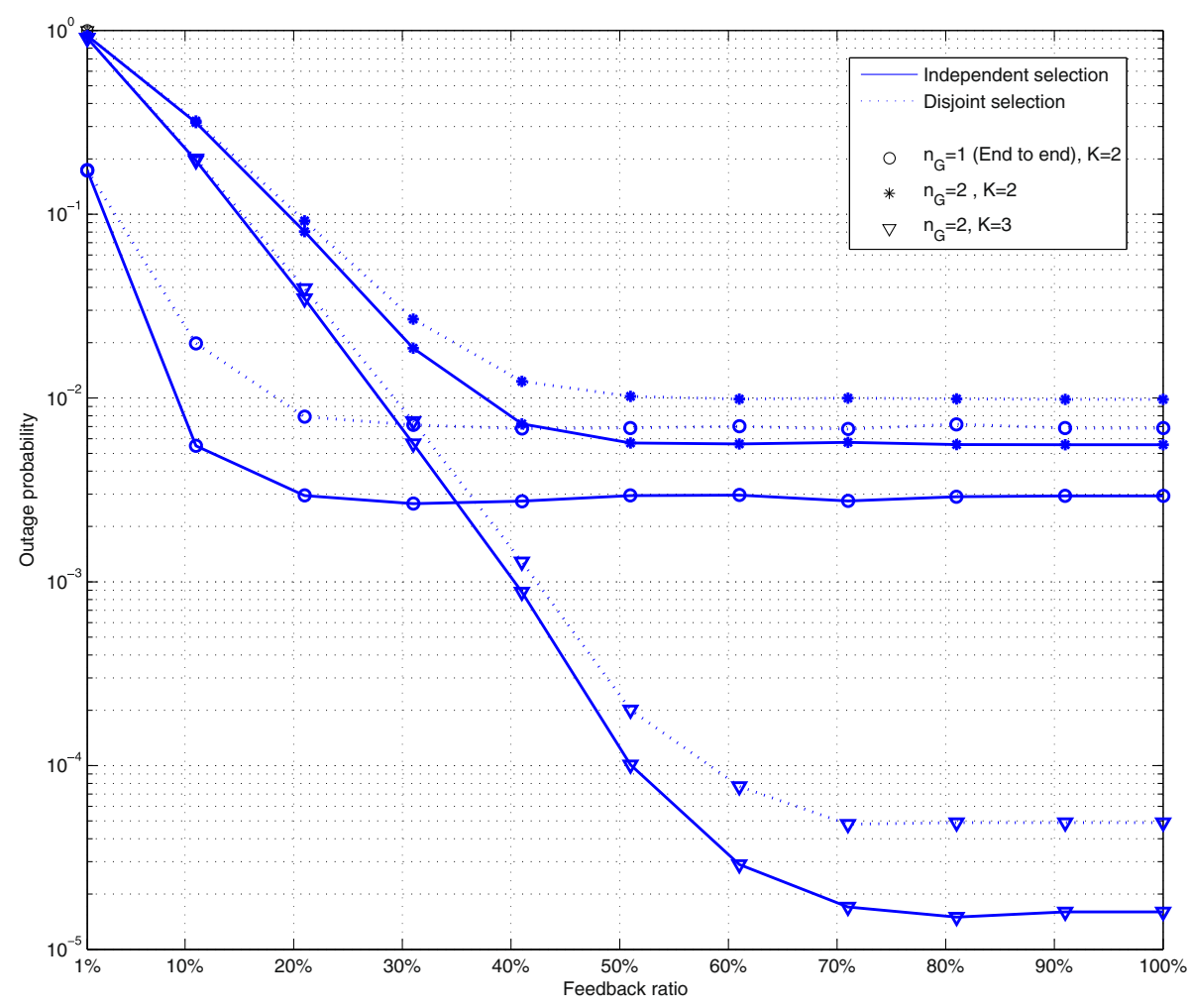

Fig. 5 The effect of the number of clusters on the outage performance 
fact, the ES technique only determines which $K$ relays should be used unlike the proposed scheme which computes also the best gain factor for each relay. Hence, we conclude that the proposed model outperforms ES in terms of outage probability in addition to the computation complexity which is reduced from $C_{E t E-I}^{E S}=O\left(N^{K}\right)$ to $C_{E t E-I}^{P}=O\left(K^{2} N^{M-1}\right)$.

Concerning SDF technique with and without power control, there is no exact expression provided for the computation complexity. However, from [29], we deduce that they are linear in function of $N$. Hence, the EndtoEnd OMP-based scheme does not outperform SDF in terms of computation complexity. However, it can be seen in Fig. 4 that the SDF scheme is outperformed by the proposed scheme in terms of outage probability. When power control is used, the performance of SDF is improved but it is still outperformed by the OMP algorithm. In fact, in addition to the gain achieved by the OMP, the performance gap between SDF and the proposed algorithm is increased due to the two-by-two hops selection process in SDF unlike the EndtoEnd OMP scheme where the selection process is done from end to end.

Figure 5 presents the effect of the number of clusters on the outage performance of different techniques $(N=$ $\left.8, K=2, N_{\mathrm{cpg}}=3, N_{c}=3,5,7\right)$.
Note that the transmitted signal is decoded and forwarded at each hop. Hence, increasing the number of hops increases the chances of incorrect decoding in the intermediate relays. Hence, as it can be seen in Fig. 5, the larger the number of hops, the higher the outage probability [34].

In addition, compared to the independent selection schemes, when the dis-joint selection is applied, all the studied techniques suffer from a loss of outage performance since the set of possible selected paths is reduced due to the dis-joint selection constraint. However, this loss is compensated by a reduction of the computation complexity. Hence, a compromise between computation complexity and outage performance is achieved between independent and dis-joint selection schemes.

It can be seen also that the proposed scheme outperforms the SDF technique with and without power control independently from the number of clusters.

Figure 6 compares the BER performance of the different studied techniques. It can be seen that-like the outage probability performance measure-the proposed EndtoEnd and PerGroup selection schemes outperform ES and SDF techniques in terms of bit error rate. Also, the EndtoEnd scheme outperfoms the PerGroup in terms of BER at the price of increased amount of CSI that need to be collected and higher complexity.

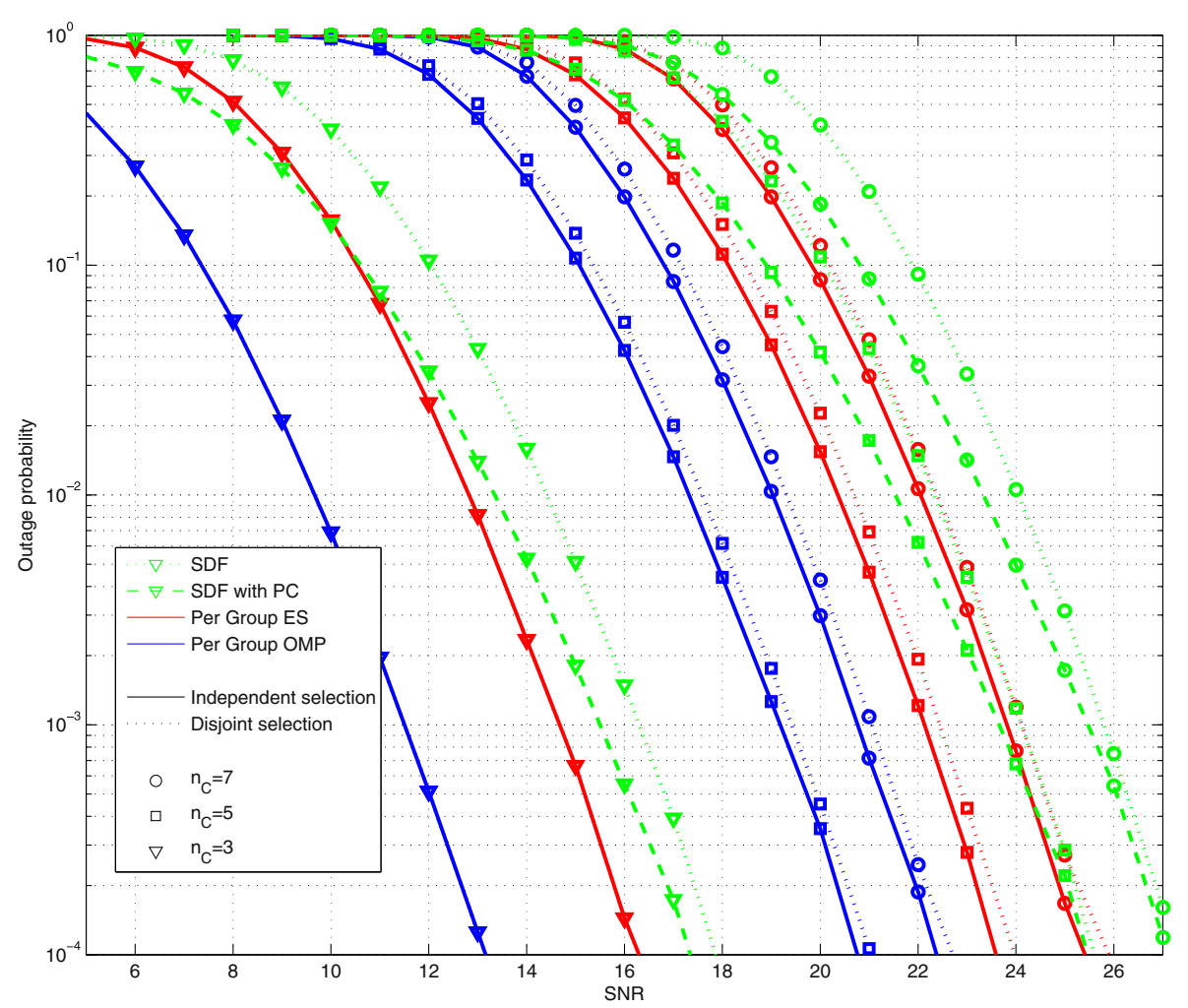

Fig. 6 Comparison of the bit error rate performance of the EndtoEnd, PerGroup, ES, and SDF techniques 


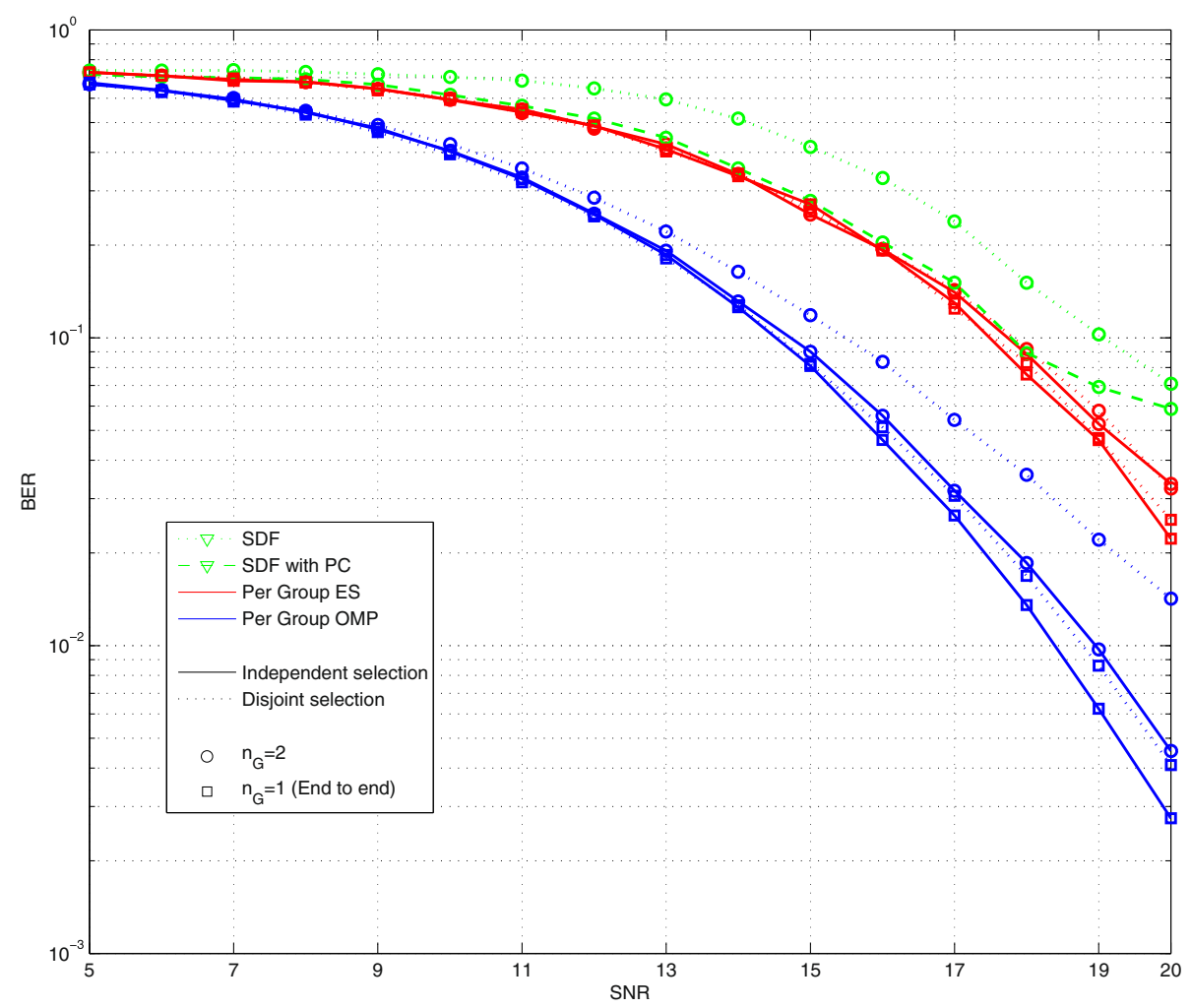

Fig. 7 The effect of the number of selected relays on the network throughput

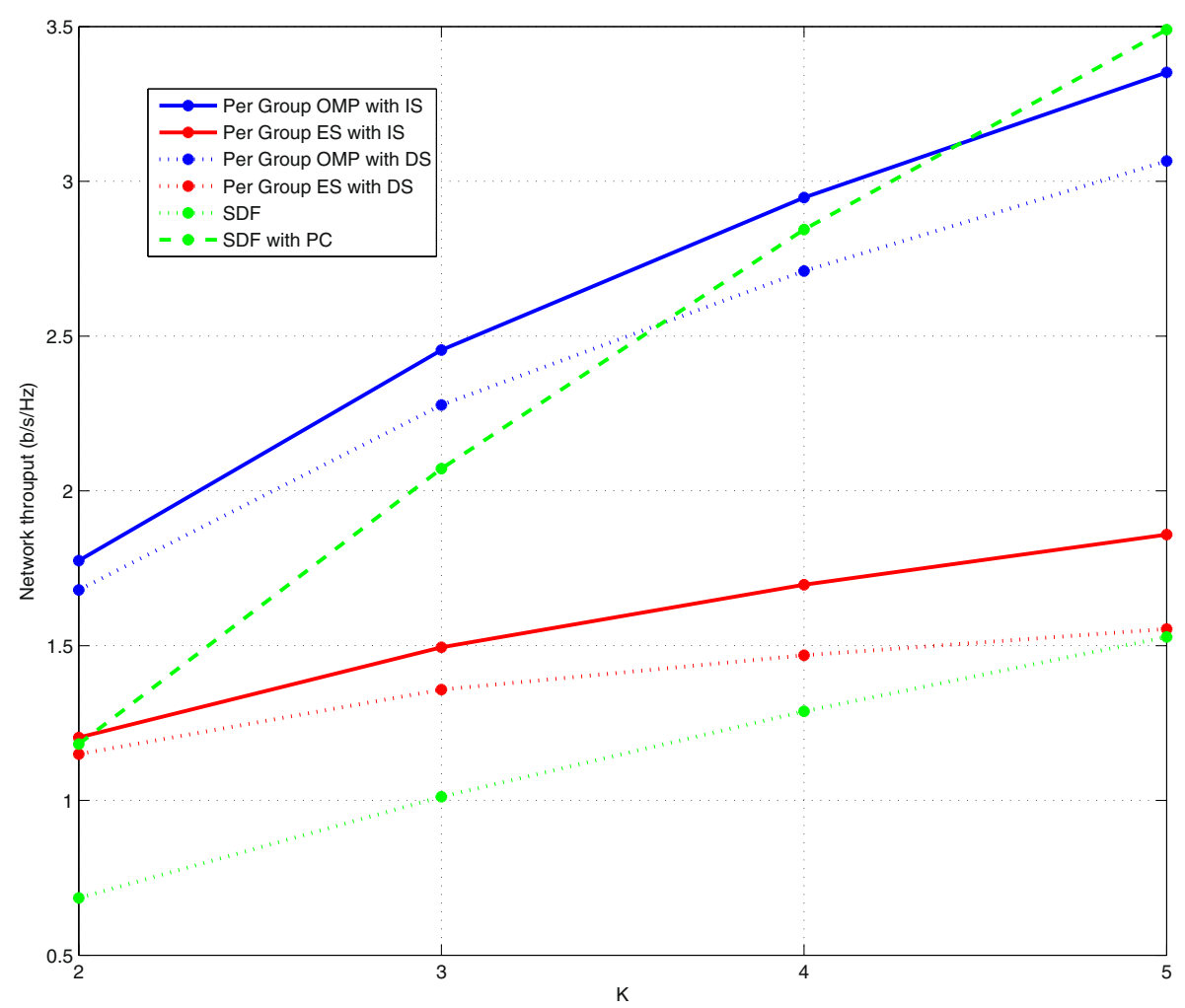

Fig. 8 The effect of the feedback percentage on the outage performance 
Figure 7 presents the effect of the number selected relays on the network throughput $\left(N=6, K=2 . .5, N_{\text {cpg }}=4\right.$, $\left.N_{c}=7\right)$. First, it can be seen that increasing the number of selected relays always improves the throughput performance because in multiple relay selection schemes and when sparse signal recovery theory is not used, it is always better to use more relays to achieve better outage performance. However, using all the relays of the network to forward the data is not practical and may lead to network congestion. Hence, the optimal $K$ is the total number of relays, but in practice, $K$ is chosen by the network administrator to make a compromise between the desired outage performance and network congestion. Also, even when selecting five relays at each hop out of six, the selection vector is still sparse since $K$ paths out of $N^{\left(N_{\mathrm{cpg}}-1\right)}$ are selected in each group. Furthermore, the outage probability is always reduced when increasing the number of relays because the sensing matrix $\mathbf{L}^{H}$ in Eq. (10) is a square full-rank ${ }^{3}$ matrix as in [17].

Note also that when increasing the number of selected relays, the proposed PerGroup technique (with $N_{\mathrm{cpg}}=4$ ) is outperformed by the SDF technique in terms of network throughput since the gain of the PerGroup schemes compared to SDF in terms of outage probability is not worth the time needed to collect all the needed CSI for the selection.
Figure 8 presents the effect of the feedback reduction on the outage probability on the PerGroup and EndtoEnd selection schemes $\left(N=10, K=2,3, N_{G}=1,2, N_{c}=5\right)$. It can be seen that when using limited knowledge of the CSI, the outage performance is almost the same for full CSI selection schemes if the feedback percentage is high enough. This feedback percentage threshold is smaller when the number of possible paths is bigger or the number of selected relays is smaller. In particular, when $\left(n_{G}=1\right.$ (EndtoEnd scheme), $K=2)$ ), the knowledge of $20 \%$ of the CSI is enough to achieve the outage performance of full CSI knowledge model. However, for models where the number of paths is lower or the number of selected relays is bigger, a higher feedback percentage is needed to achieve the full CSI selection scheme performance (40\% when $n_{G}=2, K=2$ and $70 \%$ when $n_{G}=2, K=3$ ).

In terms of network throughput, it can be seen in Fig. 9 $\left(N=10, K=2,3, N_{G}=1,2, N_{c}=5\right)$ that for both the EndtoEnd and PerGroup schemes, when the nodes feedback more than a specific percentage, the performance starts decreasing since the gain in the equivalent received SNR is no longer worth the time needed to collect the CSI. This feedback percentage threshold is bigger when the number of possible paths is larger and is not affected by the number of selected relays. Note also that for EndtoEnd scheme, the system can not be implemented

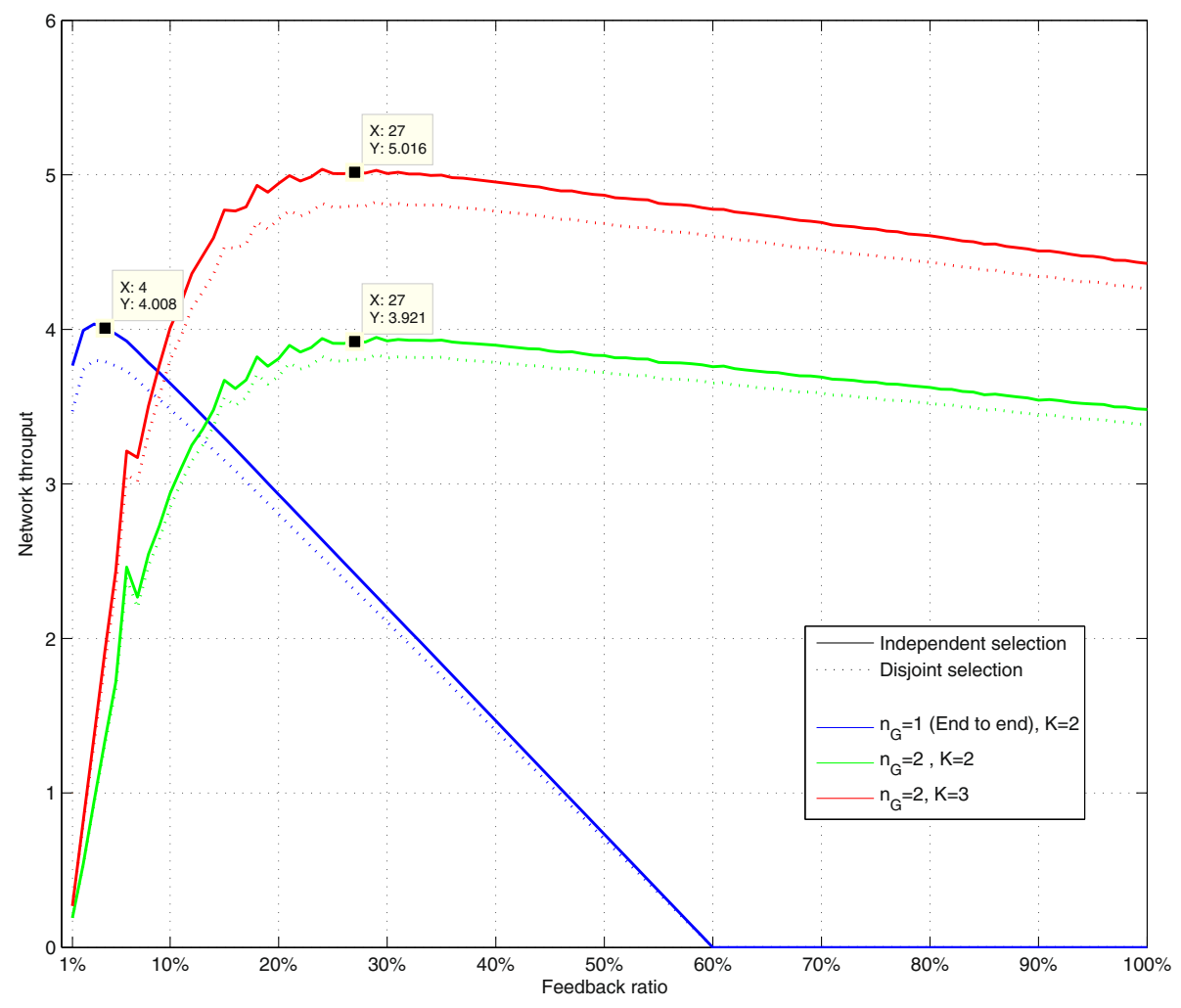

Fig. 9 The effect of the feedback percentage on the network throughput 
in practice with a feedback percentage higher than $60 \%$ since the channel would change after the source finished collecting all the CSI unless the channel coherence time is longer.

\section{Conclusions}

In this paper, two novel techniques were proposed and investigated to select multiple relays in a large multi-hop DF network based on MSE minimization. By exploiting sparse signal recovery theory, these techniques improve the outage performance compared to the ES and SDF techniques with and without power control. The computation complexity is also reduced compared to the ES technique. Furthermore, performance, feasibility, and selection complexity tradeoffs are considered between the different studied techniques and especially between the EndtoEnd and PerGroup selection schemes. Extensive simulations were also conducted to evaluate and compare the performance of the proposed techniques with the conventional ones. Finally, we demonstrated that by using the proposed limitedfeedback scheme, almost the same outage performance can be achieved while using only partial CSI knowledge of the CSI.

\section{Endnotes}

${ }^{1}$ Note that the maximum number of selected paths $K$ is $N$ (All the relays are used); and even in this case the selection vector $\mathbf{g}_{\mathbf{s}}$ remains sparse since the selection is done among the set of all possible paths.

${ }^{2} \mathrm{We}$ assume the existence of a virtual node that will combine all the received signals by the end of each group using MRC to be able to compute and optimize the MSE corresponding to that group. However, this MRC is not done in reality unless the last node of that group is the destination.

${ }^{3}$ Note that even thought the sensing matrix $L^{H}$ is fullrank and the selection problem is well-posed, the optimal solution is not sparse (all the paths are selected) and OMP is used to compute the 'best' $K$ sparse solution.

\section{Acknowledgements \\ This paper was made possible by NPRP grant 6-070-2-024 from the Qatar National Research Fund (a member of Qatar Foundation). The statements made herein are solely the responsibility of the authors.}

\section{Competing interests}

The authors declare that they have no competing interests.

\section{Author details}

${ }^{1}$ Electrical Engineering Department, Qatar University, Doha, Qatar. ${ }^{2}$ University of Burgundy, Dijon, France. ${ }^{3}$ Electrical Engineering Department, UT Dallas, Richardson, TX, USA. ${ }^{4}$ Computer Science and Engineering Department, Qatar University, Doha, Qatar.
Received: 10 October 2015 Accepted: 28 June 2016

Published online: 21 July 2016

\section{References}

1. T Cover, AE Gamal, Capacity theorems for the relay channel. IEEE Trans. Inf. Theory. 25(5), 572-584 (1979)

2. A Sendonaris, E Erkip, B Aazhang, in IEEE International Symposium on Information Theory (ISIT). Increasing uplink capacity via user cooperation diversity (Saint-Petersburg, Russia, 1998), p. 156

3. A Sendonaris, E Erkip, B Aazhang, User cooperation diversity-part I: system description. IEEE Trans. Commun. 51(11), 1927-1938 (2003)

4. A Sendonaris, E Erkip, B Aazhang, User cooperation diversity-part II: implementation aspects and performance analysis. IEEE Trans. Commun. 51(11), 1939-1948 (2003)

5. JN Laneman, DNC Tse, GW Wornell, Cooperative diversity in wireless networks: efficient protocols and outage behavior. IEEE Trans. Inf. Theory. 50(12), 3062-3080 (2004)

6. A Gouissem, R Hamila, MO Hasna, Outage performance of cooperative systems under IQ imbalance. IEEE Trans. Commun. 62(5), 1480-1489 (2014)

7. A Gouissem, MO Hasna, R Hamila, H Besbes, F Abdelkefi, in IEEE Vehicular Technology Conference (VTC Fall). Outage performance of OFDM ad-hoc routing with and without subcarrier grouping in multihop network (Quebec, Canada, 2012), pp. 1-5

8. A Gouissem, MO Hasna, R Hamila, H Besbes, F Abdelkefi, in IEEE 23rd International Symposium on Personal Indoor and Mobile Radio Communications (PIMRC). Optimized selective OFDMA in multihop network, (Sydney, Austraila, 2012), pp. 1348-1353

9. A Gouissem, R Hamila, MO Hasna, in IEEE Global Information Infrastructure Symposium (GIIS). Outage performance of OFDM-based relaying systems under IQ imbalance, (Trento, Italy, 2013), pp. 1-7

10. B Karakaya, HU Sokun, M Uysal, MO Hasna, in 20th IEEE Signal Processing and Communications Applications Conference (SIU). Cooperative OFDM systems for UWAC with SNR-based relay selection schemes, (Mugla, Turkey, 2012), pp. 1-4

11. SI Hussain, M-S Alouini, MO Hasna, A diversity compression and combining technique based on channel shortening for cooperative networks. IEEE Trans. Wirel. Commun. 11(2), 659-667 (2012)

12. Y Jing, $\mathrm{H}$ Jafarkhani, Single and multiple relay selection schemes and their achievable diversity orders. IEEE Trans. Wirel. Commun. 8(3), 1414-1423 (2009)

13. Y Hu, KH Li, KC Teh, An efficient successive relaying protocol for multiple-relay cooperative networks. IEEE Trans. Wirel. Commun. 11(5), 1892-1899 (2012)

14. R Ananthapadmanabha, BS Manoj, CSR Murthy, in 12th IEEE International Symposium on Personal, Indoor and Mobile Radio Communications (PIMRC). Multi-hop cellular networks: the architecture and routing protocols, vol. 2 (San diego, USA, 2001), pp. G-78-G-82

15. A Agarwal, P Kumar, in ACM Special Interest Group on Data Communications (SIGCOMM). Capacity bounds for ad hoc and hybrid wireless networks, vol. 34, No. 3, (Portland, 2004), pp. 71-81

16. O Dousse, P Thiran, M Hasler, in Twenty-First Annual Joint Conference of the IEEE Computer and Communications Societies (INFOCOM). Connectivity in ad-hoc and hybrid networks, vol. 2, (New York, USA, 2002), pp. 1079-1088

17. Junho Lee, N Al-Dhahir, Exploiting sparsity for multiple relay selection with relay gain control in large af relay networks. IEEE Wireless Commun. Lett. 2(3), 347-350 (2013)

18. J Boyer, DD Falconer, $\mathrm{H}$ Yanikomeroglu, Multihop diversity in wireless relaying channels. IEEE Trans. Commun. 52(10), 1820-1830 (2004)

19. H Li, M Lott, M Weckerle, W Zirwas, E Schulz, in The 13th IEEE International Symposium on Personal, Indoor and Mobile Radio Communications (PIMRC). Multihop communications in future mobile radio networks, vol. 1, (Lisbona, Portugal, 2002), pp. 54-58

20. Multihop diversity in wireless relaying channels. IEEE Trans. Commun. 52(9), 1605-1605 (2004)

21. AEl Shafie, N Al-Dhahir, R Hamila, A sparsity-aware cooperative protocol for cognitive radio networks with energy-harvesting primary user. IEEE Trans. Commun. 63(9), 3118-3131 (2015)

22. AO Al-Abbasi, R Hamila, WU Bajwa, N Al-Dhahir, in 2015 IEEE Global Conference on Signal and Information Processing (GlobalSIP). A general 
framework for the design and analysis of sparse fir linear equalizers, (Florida, USA, 2015), pp. 834-838

23. L Wang, G Zhang, G Wei, in 2011 6th International ICST Conference on Communications and Networking (CHINACOM). Relay selection with noisy limited feedback, (Harbin, China, 2011), pp. 400-404

24. K Elkhalil, ME Eltayeb, H Shibli, HR Bahrami, TY Al-Naffouri, in 2014 IEEE Global Communications Conference (GLOBECOM). Opportunistic relay selection in multicast relay networks using compressive sensing, (Austin, USA, 2014), pp. 3126-3131

25. ME Eltayeb, K Elkhalil, HR Bahrami, TY Al-Naffouri, Opportunistic relay selection with limited feedback. IEEE Trans. Commun. 63(8), 2885-2898 (2015)

26. KElkhalil, ME Eltayeb, A Kammoun, TY Al-Naffouri, HR Bahrami, On the feedback reduction of relay aided multiuser networks using compressive sensing. archived. http://arxiv.org/pdf/1505.00582.pdf Accessed 4 May 2015

27. Z Wang, $W X u, J$ Lin, in 2014 IEEE International Symposium on Wireless Personal Multimedia Communications (WPMC). Compressive cooperative schemes with multiple relays, (Sydney, Australia, 2014), pp. 536-540

28. JA Tropp, AC Gilbert, Signal recovery from random measurements via orthogonal matching pursuit. IEEE Trans. Inf. Theory. 53(12), 4655-4666 (2007)

29. $Y$ Zhang, $Y X u, Y$ Cai, in 2nd, IEEE International Conference on Signal Processing and Communication Systems (ICSPCS). Relay selection utilizing power control for decode-and-forward wireless relay networks (IEEE, Gold Coast, Australia, 2008), pp. 1-5

30. B Lin, PH Ho, LL Xie, X Shen, J Tapolcai, Optimal relay station placement in broadband wireless access networks. IEEE Trans. Mobile Comput. 9(2), 259-269 (2010)

31. MR Souryal, BR Vojcic, in Proceedings. of IEEE International Conference on Acoustics, Speech and Signal Processing, ICASSP. Performance of amplify-and-forward and decode-and-forward relaying in Rayleigh fading with turbo codes, vol. 4, (Toulouse, France, 2006), pp. IV-IV

32. ST Qaseem, TY AI-Naffouri, in IEEE International Conference on Communications (ICC). Compressive sensing for reducing feedback in MIMO broadcast channels, (Cape Town, South Africa, 2010), pp. 1-5

33. ME Eltayeb, TY Al-Naffouri, HR Bahrami, Compressive sensing for feedback reduction in MIMO broadcast channels. IEEE Trans. Commun. 62(9), 3209-3222 (2014)

34. B Gui, L Dai, L Cimini, Routing strategies in multihop cooperative networks. IEEE Trans. Wirel. Commun. 8(2), 843-855 (2009)

\section{Submit your manuscript to a SpringerOpen ${ }^{\circ}$ journal and benefit from:}

- Convenient online submission

- Rigorous peer review

- Immediate publication on acceptance

- Open access: articles freely available online

- High visibility within the field

- Retaining the copyright to your article

Submit your next manuscript at $\gg$ springeropen.com 